\title{
Leveraging Icebreaking Tasks to Facilitate Uptake of Voice Communication in Multiplayer Games
}

\author{
Kieran Hicks ${ }^{1}$, Kathrin Gerling ${ }^{2}$, Patrick Dickinson ${ }^{1}$, Conor Linehan ${ }^{3}$, Carl Gowen ${ }^{1}$ \\ ${ }^{1}$ University of Lincoln, UK \\ ${ }^{2}$ KU Leuven, Belgium \\ ${ }^{3}$ University College Cork, Ireland \\ khi cks@i ncol n. ac. uk
}

\begin{abstract}
Voice Communication (VC) is widely employed by developers as an essential component of online games. Typically, it is assumed that communications through this mechanism will be helpful and enjoyable, but existing literature suggests that the entry into VC can be problematic. In this paper, we present a study that attempts to mitigate player discomfort when first engaging with VC with strangers, through the use of traditional icebreaking tasks. We integrate these into the game RET, an online cooperative first person shooter which requires effective communication for players to succeed. An online user study with 18 participants suggests that icebreaking tasks can contribute to a positive VC experience, but their inclusion also creates further issues to be considered for successful integration.
\end{abstract}

Keywords: Voice communication, player experience, game design.

\section{Introduction}

Voice communication (VC) is increasingly seen as a standard feature in online multiplayer gaming, e.g. Overwatch [4], The Division [37] and, Destiny [7]. A small body of research on the topic suggests that, on the one hand, VC may promote a feeling of connection between players, and facilitates coordinated play [42]; on the other hand, VC can make players feel more vulnerable and open to new types of antisocial behaviour $[41,40]$. Given the increased popularity of online multiplayer modes, the integration of $\mathrm{VC}$ as the default method for player coordination in contemporary games, but many of the initially reported issues still remain [42], suggesting a need for game designers and researchers to develop ways of facilitating positive VC experiences.

In our paper, we address this issue by leveraging traditional icebreaking tasks which are small group based tasks designed to introduce people to each other and encourage conversation. We use these to support the on-boarding phase of games, as this is a key element in retaining players [32]. We present $R E T$, a three-player online game in which the successful completion of team challenges requires effective communication between players. In a study with 18 participants, we explore the impact of 
in-game icebreaking tasks on player experience, and perceptions of VC. This was achieved through a study using post-hoc surveys and interviews. Our results show that icebreaking tasks are generally effective in bringing players together, but that adaptations are necessary to seamlessly integrate them into games and ensure adequate replayability. Building on these findings, we discuss strategies for the development of in-game icebreaking tasks to facilitate entry into $\mathrm{VC}$, and discuss the wider implications that $\mathrm{VC}$ has for player experience.

This paper makes the two main contributions: 1) We present one approach to reducing player discomfort when first engaging with $\mathrm{VC}$, through the design and evaluation of in-game icebreaking tasks. 2) We discuss issues surrounding the wider integration of icebreakers into online multiplayer games.

\section{Background}

We provide an overview of previous work on the use of $\mathrm{VC}$ in games along with insights into icebreaking tasks to facilitate social interaction in gaming settings and beyond.

\subsection{Voice Communication in Games}

Since the late 1990s, VC has been used as an alternative to text chat in online games. It was first used for back channel communication through third-party voiceover-IP software, such as TeamSpeak [31]. More recently, VC has been built directly into games; for example, in the Xbox LIVE online multiplayer platform [40]. In early online games, voice chat was sought frequently in competitive team-based firstperson shooter (FPS) games to support fast-paced combat [40]. Likewise, in the massively multiplayer online (MMO) genre, strategic advantage was a primary factor that increased the uptake of VC [40, 44]. Recently some games, such as DayZ [8] have made use of the voice communication as a game mechanic, through an in-game proximity system that only allows nearby players in the game world to communicate. [10]. Voice has also been used as a method for control in single player games [9].

Previous work has broadly explored player motives to engage in VC. Beyond using it to gain competitive advantage, some players use online gaming not just to connect with their teammates and opponents, but also as a way of staying in touch with friends [44], and research suggests that gaming communities may form for both social and cooperative reasons [45]. Within these communities, players may receive social and emotional support [33] and there is evidence that voice communication in games has positive social effects; for example, a reduction of loneliness among players [44]. In this context, research suggests that $\mathrm{VC}$ conveys more information about the communicator than text [40], and may therefore establish increased trust between conversation partners [6].

Wadley et al. [40] found that players considered voice to have greater emotional impact than text, with profound implications for player experience. For example, one rude player can demoralise the rest of the team [40]. Previous research also identified 
concerns regarding anxiety and harassment that were detrimental to players' experiences of VC $[39,40,41]$. Other problematic aspects include player behaviour that adds unnecessary noise to the voice channel e.g. television programs being picked up by the microphone [17], and technical difficulties especially when using third-party tools [39].

\subsection{Icebreakers to Facilitate Social Interaction}

Icebreakers have been long used as a method of familiarising strangers in small groups to increase collaboration and encourage positive communication. For example, they are applied to introduce groups of adult learners [12], or as a means to initiate conversation in public spaces [26]. Icebreakers have also been found to be a method of supporting increased collaboration and aiding in the development of online communities [14], e.g., in an emerging online learning community users were asked to name a film character that they identified with, revealing a piece of personal information whilst encouraging discussion [14]. Different types of icebreaking tasks can be employed depending on the nature of the existing relationship between participants, situations in which the participants will be strangers, tasks that facilitate introductions and getting to know each other are successful [43] e.g. asking a group to sort into alphabetical order encourages introductions $[42,43]$.

Interestingly, games are often applied as icebreaking tasks [26], rather than being viewed as a setting that could benefit from icebreaking activities. For example, Jarusriboonchai et al. [21] leveraged a co-located game that involved players asking simple questions as an introductory icebreaking task. Generally, research suggests that games can be more effective than traditional icebreaking tasks, highlighting the benefits of a playful setting [11].

\section{RET: A System to Study Icebreaking Tasks in Games}

In order to study the impact of icebreaking tasks to facilitate $\mathrm{VC}$ in online gaming, we created RET, a co-operative online game. The goal of RET is to progress through a series of challenges that vary in activity such as jumping on platforms and navigating mazes (See Figure 1). The game is played with three players; each is given either the Healer, Tank or Damage role at random upon starting the game. These three roles each have a different ability requiring players to work together. RET was implemented using the Unity3D engine [38] and was distributed via the game marketplace itch.io.

\subsection{Gameplay}

RET is a first person shooter to be played with three persons that features a total of nine puzzles (see Figure 3 ) that must be solved co-operatively and require players to both discuss tactics and swap between roles (e.g., the role of Healer must be swapped between players to heal each other). The Tank role allows the player to raise a large 
shield that can be used to defend both themselves and fellow players, The Damage role is able to shoot and destroy enemies that exist through the level whilst also having a higher jump to reach certain platforms, and the Healer role is granted the ability to shoot orbs that heal fellow players. There are some instances in which the game uses distributed information across players, e.g., a player cannot see their own health value and instead must rely on other players to keep them informed (See Figure 2).

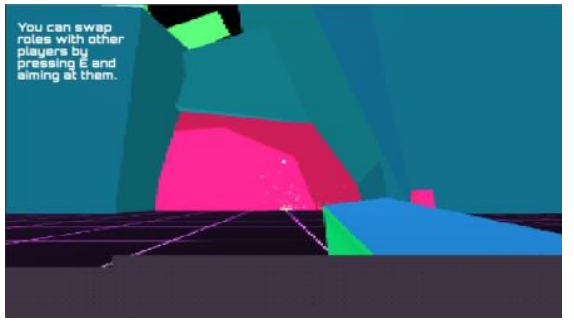

Fig. 1: Gameplay view of RET.

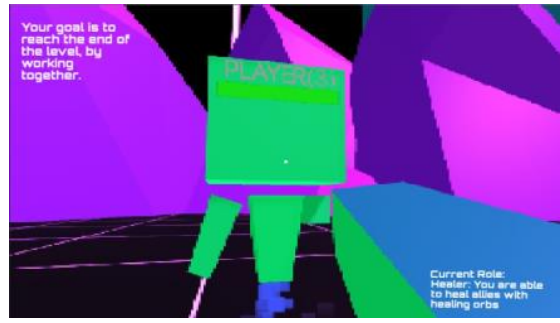

Fig. 2: Observing the health of a teammate.

Controls. Players can navigate the environment using the WASD keys to move, space to jump, and the mouse to orientate and look around. Unique abilities associated with roles can be accessed through the left mouse click. The players' avatar is able to interact with certain objects in the environment by pressing the F key, and each player can request to change roles with other players.

Voice Communication. As RET implements information sharing as a core mechanic, it lends itself to voice communication to facilitate this process. The game features an in-game voice communication solution implemented via an open channel with an always on microphone (i.e., continuous transmission). A text-based chat channel is also included.

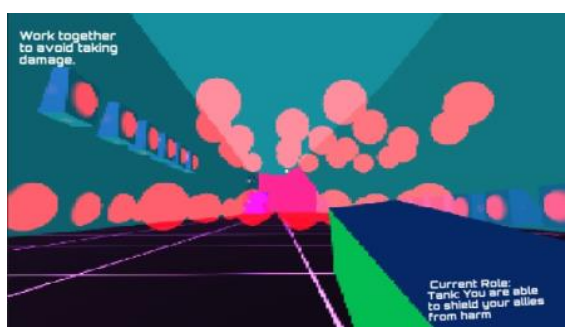

Fig. 3: Puzzle solving.

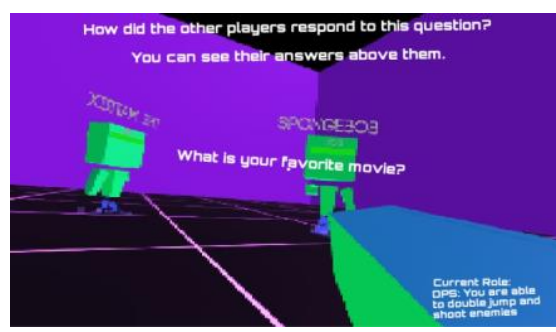

Fig. 4: Engaging with the icebreaking task.

\subsection{Integration of Icebreaking Tasks}

Traditional icebreaking tasks prominently feature information exchange, e.g., users are tasked with arranging the group into alphabetical order which prompts participants to ask each other's names or participants are posed with a statement such as "what $x$ items could you not live without" [43]. RET builds on these previously successful icebreaking tasks [15] and uses simple questions to encourage players to interact with 
each other (See Figure 4). The task is situated within the game world and takes about 90 seconds to complete. When first joining the game, players are prompted to answer several questions e.g., What is your favourite hobby?, What is your favourite movie?, Where are you from?, Current favourite game?. Once all players have completed this part, a prompt appears that introduces the team and encourages players to greet each other by speaking their nickname displayed on each player's avatar. After 30 seconds, answers are shared with the group by displaying them above each avatar. The game then encourages players to discuss; however, to see answers, players need to be in close proximity in the virtual space. Therefore, they do not just have to engage in verbal communication but also interact through physical location of their avatars. This process is repeated three times, then the main game begins by teleporting all players into the starting area of the first level.

\section{Study: Exploring the Effects of Icebreaking Tasks in Multiplayer Games}

Here, we report results of a study we carried out to gain insights into both player perceptions of the game RET and the integration of icebreaking tasks to facilitate engagement with VC.

\subsection{Research Questions}

We aim to answer two main questions related to the integration of in-game icebreaking tasks in RET, (1) what effect does the inclusion of these icebreaking tasks have on the player experience of $R E T$ and (2) if the icebreaking tasks had any effect on reducing player perceived discomfort in using $\mathrm{VC}$ with strangers.

\subsection{Measures}

In our study, we use post-game questionnaires and interviews to gain insights into player experience, combining quantitative and qualitative data.

Questionnaires. We made use of several standard questionnaires and a custom post-game questionnaire. This was done through an in-game survey approach as Frommel et al. found that integrated questionnaires can help minimise gameplay interruptions [16]. The first few questions focused on demographic information. Following this, participants were asked to rate several statements on a seven-point Likert scale, for example, "I enjoyed the communication method" and "I felt comfortable using the communication method". This part of our study focused on participant enjoyment and feelings toward VC. Finally, participants were asked to fill in the Player Experience and Needs Satisfaction (PENS) Questionnaire [27] as this has previously been applied in games research [3]. Through using the PENS questionnaire after each condition, we gained insight into how the icebreaking task affected the player experience. When participants had played through all three conditions, they were asked to 
rate each condition on a Likert scale between $1-7$ where 1 is "Dislike" and 7 is "Like".

Interviews. After the play session was complete, participants were invited to take part in a short one-on-one open interview. This interview was semi-structured with several questions that all participants were asked surrounding feelings of discomfort and shyness and what effects they felt the icebreaker had on the game. At the end of the interview, participants were given an opportunity to ask questions relating to the research.

\subsection{Participants and Procedure}

We recruited 18 participants through social media including Facebook, Twitter, and an online learning support system at <Removed for Review> ( 1 female, average age $22, \mathrm{SD}=3.42$ ). Participants took part in groups of three, each session lasted around 1.5 hours. Participants were compensated with a $£ 10$ Amazon voucher. The research was approved by the ethics board at <removed for blind review> and all participants gave informed consent.

All participants took part remotely to accurately simulate the context of online multiplayer gaming. At the start of the session, participants were then given a brief overview of the game RET and the general procedure. We followed a within-subjects design (counterbalanced using Latin square) with all participants experiencing three conditions: VC but no icebreaking task, VC and icebreaking task, Icebreaking task but no VC. After playing through each condition, participants were asked to complete the integrated post-play survey. At the end of the study, participants were asked to rate the three conditions, and participate in a closing interview.

\subsection{Quantitative Results}

Data were analyzed using Friedman's ANOVA, and we followed up with Wilcoxon signed-rank tests for pairwise comparisons. Results for the initial post-game statements reveal that of the three communication methods, participants prefer the inclusion of $\left(X^{2}(2)=19.097, p<.05\right)$. Participants enjoyed VC over text (text $\mathrm{M}=2.88$, $\mathrm{SD}=1.91, \mathrm{VC} \mathrm{M}=6.00, \mathrm{SD}=1.15 ; \mathrm{Z}=-3.224, \mathrm{p}=0.001)$. There was no significant difference in enjoyment between voice with and without icebreakers. Though slightly higher feelings of comfort were reported (without icebreaker $\mathrm{M}=5.66, \mathrm{SD}=1.63$, with icebreaker $\mathrm{M}=5.83, \mathrm{SD}=1.60$ ) the difference was not significant.

Results for the PENS questionnaire show that RET provided players with a generally positive player experience (see Figure 5). In terms of perceived competence, we found significant differences between all conditions $\left(X^{2}(2)=7.969, p<.05\right)$. Pairwise comparisons revealed no significant difference in perceived competence in the voice communication conditions. There was a significant difference for relatedness $\left(X^{2}(2)\right.$ $=8.853, p<.05)$, pairwise comparisons showed a significant difference between conditions with voice and without $(\mathrm{Z}=-2.180, \mathrm{p}=0.029)$, but not between voice with icebreaker and voice without icebreaker. 


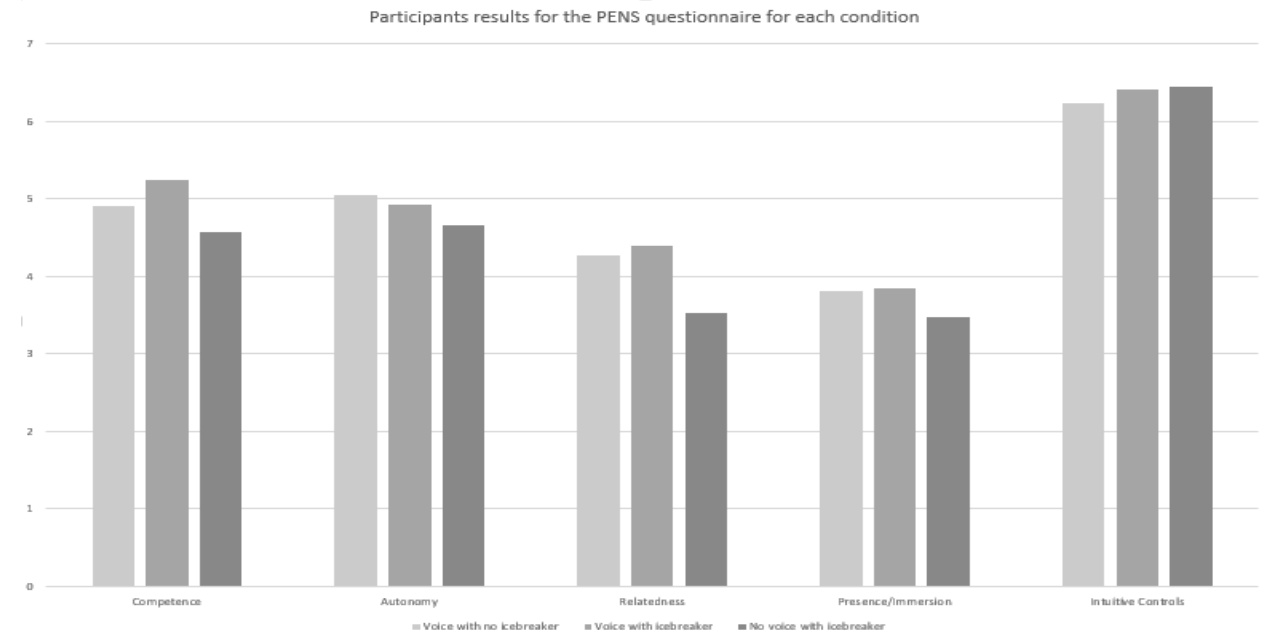

Fig. 5: PENS [27] results for RET (7=strongly agree, $1=$ strongly disagree).

\subsection{Data Analysis}

Interview data were analysed using a Thematic Analysis approach according to Braun and Clarke [2]. Responses were coded, and descriptive category codes were applied to 486 data points with a total of 31 categories emerging (See Figure 6).

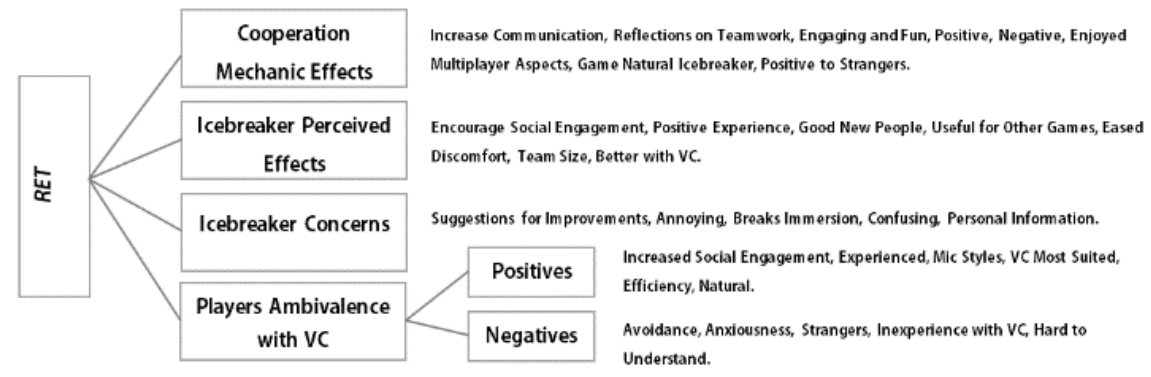

Fig. 6: Breakdown of themes emerging from interviews.

\subsection{Qualitative Results}

Our results reveal four key themes, Benefits of Icebreaking Tasks (89 occurrences), Negative Effects of Icebreaking Tasks (77 occurrences), Effects of Cooperative Mechanics (176 occurrences), and Players' Ambivalent Perspectives on VC (131 occurrences) which is comprised of two subthemes Positives of VC (70 occurrences) and Negatives of VC (61 occurrences). 
Theme 1: Benefits of Icebreaking Tasks

This theme emerged around the perceived benefits that the icebreaking task provided, focusing on two aspects; its effects on social engagement through the encouragement of conversation and relieving feelings of discomfort, but also its effects on increasing the overall experience by enhancing collaboration.

Encouraging social engagement between players. Results surrounding this theme were frequently about the effects that the icebreaker had on player social engagement, the icebreaking tasks were reported to initiate conversations between players by encouraging them to talk about their answers e.g. "The ice breaker certainly helped in getting to know the other players [...]". One aspect that arose during this was participants responding with humorous answers to the questions which then would break the ice allowing people to laugh and prompt discussion. Participants also expressed that the inclusion of the icebreaker questions about personal information allowed them to feel more connected to other players, e.g., "I really liked it, it made me feel more connected to the players". Another sentiment that was expressed was of the icebreaker allowing the player to see their fellow players as real humans "[...] it felt like they were more real, if that makes sense, like I had already dived further into who they were". This sentiment occurred frequently e.g. "I have to admit it did kinda humanise everyone if that makes sense as it was a bit comforting." These results reveal that by learning and sharing a piece of personal information it facilitated players to feel more connected and to humanise each other.

Effect of Icebreakers on Player Experience. Responses in this theme focus on the ways in which the icebreaker had an effect on player experience beyond social engagement. Participants stated that they were more inclined to communicate openly with other players after participating in the icebreaking tasks. Additionally, they suggested icebreakers enabled them to communicate more efficiently. Participants also reported how they would like to see the icebreaking task in other games, discussing what potential benefits it could enable. Additionally, participants reflected again on the humanising nature of icebreakers discussed in the previous theme e.g., "team based games like MOBA's may benefit from this as there is a lot of anger and hate thrown around in a match and having this information may be a way to make people think that there is another person behind the screen". Lastly, participants felt the benefits of the icebreaking tasks were more pronounced when using VC over text-based communication.

\section{Theme 2: Negative Effects of Icebreaking Tasks}

This theme addresses concerns that participants voiced regarding the inclusion of icebreaking tasks. It explores issues relating to repeated and continued use, along with perspectives on personal information sharing.

Players' Concerns. Results show that participants had ambivalent thoughts on the inclusion of icebreaking tasks. While clearly acknowledging the benefits that the tasks provided, players also highlighted the risk that participation in icebreakers might be- 
come burdensome over time. Participants suggested that it would be important that players were given an opt-out. Some participants further suggested that icebreakers could interfere with players' immersion, breaking the context of the game if icebreakers were not directly embedded into play, for example stating that "they were somewhat detrimental to the experience as it took the attention away from the game that the players were wanting to play.". Participants also found the inclusion of the icebreaking task confusing as it was not something they were used to in games. Lastly, participants considered the implementation of the icebreaker problematic as it required them to share personal information with strangers, introducing the risk of vulnerability if they for example revealed their gender.

Suggestions for Improvement. Throughout the analysis, participants frequently expressed ideas, opinions, and suggestions on how the icebreakers could be modified depending on the game, genre or group of players participating. The suggestions in this theme ranged from large changes such as all players having a small biography shared with other players, and small scale suggestions such as lengthening or shortening the amount of time between each round of icebreaking tasks. Participants also expressed the desire to increase integration of icebreaking tasks, directly linking information exchange with play (e.g., implementing "a large door with the word "UP" can only be opened by the player who gave this as one of his answers"). In contrast, other participants suggested playing icebreaking tasks during natural breaks of play, for example, to cover waiting times when the game was loading.

\section{Theme 3: Effects of Cooperative Mechanics}

This theme details participants' reflections of the effects of cooperative mechanics on player experience. In particular, codes that emerged in this theme address effects on communication, reflections on teamwork and aspects of the design that participants either enjoyed or disliked.

Mechanics encouraging communication. Participants expressed the effect that the forced cooperation elements and the role-based nature of the game had on communication between players. Many participants felt that the inclusion of such mechanics facilitated and encouraged communication. Additionally, participants reported that role swapping and hidden information also encouraged communication, for example stating that "Instead of simply accepting who is whom, we had to find out ourself with the voice/text and help each other out as much as possible." While they realised that game mechanics increased communication, participants were also quick to point out possible positives and negatives that could emerge from them. For example, one participant expressed dismay having died several times, not having judged their (invisible) remaining health correctly, and finding it too difficult to communicate with other players to receive accurate information within good time.

Player reflections on teamwork and collaboration. Participants shared reflections on the gameplay experience surrounding teamwork and the sense of perceived fellowship which emerged from the inclusion of the communication mechanics in addition to the differing communication methods of $\mathrm{VC}$ and text. The results that fall into this category generally comprise participants' recollections of specific moments where they were required to use communication, for example, one player outlined that "after 
going through the barriers as medic I had to ask my team what my health was and whether I need to heal". Participants also reported that the game mechanics acted as a natural icebreaker by facilitating a common means for communication among fellow players, as the game provides a reason to communicate. Participants expressed feeling less anxious initially speaking, as conversations already were ongoing.

\section{Theme 4: Players' Ambivalent Perspectives on Voice Communication}

Player perspectives on the general inclusion of $\mathrm{VC}$ in video games were ambivalent. This theme is split into two sub-themes; the player perceived positives and negatives.

Positives of voice communication. The most frequently occurring node in this sub-theme was on the effects of VC on social engagement; participants expressed that they felt the communication was much more natural and likely to occur when using VC due to the more efficient and personal nature. Participants also expressed their opinions on the specific implementation of VC that we chose for RET (open mic, always on). Some participants considered the open mic a great feature that reduced discomfort as it made communication more easily accessible, with one participant commenting that "I prefer to just be able to talk, every time I have to push a button to talk it distracts me from the game and ruins my immersion".

Negatives of voice communication. Participants also reflected on the negative side of VC. For example, participants frequently stated that they would avoid using VC due to fears of harassment regarding gender, or that they may speak English as a second language. Tying into this, participants also revealed that they would try to avoid using VC entirely with strangers and instead just use it with friends or a mixture between the two, for example, one participant stating that "[..] I would never go in blind so to speak and just start talking to strangers". Participants also stated that inexperience using VC as a cause for lack of engagement. Lastly, participants noted that VC was sometimes hard to understand due to low volume or internet quality, creating awkward situations (e.g., asking someone to repeat themselves several times).

\section{Discussion}

In this paper, we outline challenges and opportunities associated with the use of VC, and present RET, a game that leverages traditional icebreaking tasks to facilitate VC. Our results demonstrate that icebreaking tasks can contribute to a positive VC experience, but also show that additional considerations are necessary to optimally integrate icebreaking tasks into playful environments. Here, we discuss strategies for in-game icebreaker design, and we reflect on the wider implications of the increasing use of $\mathrm{VC}$ in games.

\subsection{The Effects of Icebreaking Tasks on Player Experience}

Players experience awkwardness when speaking to strangers that icebreaking tasks can help reduce, creating a more positive experience by providing increased oppor- 
tunity for communication. However, that does not mean that icebreakers are without risk; based on the results of our thematic analysis, there are issues which may negatively impact the player experience, if not confronted. The impact that in-game icebreaking tasks have is dependent on the design and implementation of the task. If integrated with care, as outlined in the following section, there is opportunity for player experience to be improved. Poor implementation such as repetitive or overly personal tasks will have a negative effect on not just the icebreaker experience but the game as a whole. Additionally, it is important to keep in mind that quantitative results regarding player experience suggest that icebreaking tasks had no effect on how the game was generally perceived, suggesting that although players may experience discomfort in the onboarding phase, this does not translate into a generally negative player experience.

\subsection{Adapting Icebreaking Tasks to Games}

Our results show that traditional icebreaking tasks need to be further developed to be fully suitable for gaming environments. Here we will discuss the most important aspects for improvement and adaption to player feedback.

Appropriate Content for In-Game Icebreaking Tasks: Personal Preferences Rather Than Personal Information

Traditionally, icebreaking questions encourage individuals to share personal information. For example, in RET we included a question that prompted players to state where they are from. Our results suggest that the revealing nature of voice communication already requires players to share more personal information than they may be comfortable with. Hence, when directly involving players in communication, designers should avoid asking players to reveal information that could expose them to harassment directly targeting aspects of their personality (see Study 1). There is a balancing act in the design of these questions, as they also serve as the foundation for players getting to know each other. Instead of asking player to reveal personal information, we therefore suggest designers focus on perhaps less vulnerable personal preferences. For example, a question such as 'What is your favourite movie?' allows a player to express an aspect of their person without revealing substantial personal information. This raises the issue of how to deal with the repetitive nature of in-game icebreaking tasks. When wishing to follow a question-answer format, it is therefore important to consider other sources of information relevant to play, for example leveraging performance data (e.g., weapon usage, most helpful player etc.) to inform the generation of questions tying the icebreaker further into the overall experience.

Exemplary game mechanic: Sharing preferences could be implemented in a fighting game before the round countdown. For example, a statement such as "I like character X the most" could appear and prompt players to rate it between 1 to 5 with 5 being "Love it" and 1 being "Don't agree". Values could be assigned to controller buttons, and when both players have rated the statement their answers would appear on the screen for discussion. This avoids requiring the player to reveal personal information but still allows personality to show through character preferences. 
Implementation of In-Game Icebreaking Tasks: Integrating Solutions Into the Main Game

Icebreaking tasks in traditional environments are often presented as standalone activities facilitated by a moderator. The implementation we chose in RET was similar. However, while this approach was generally effective, there were concerns around the extra activity breaking immersion and flow of the game. To address this issue, we suggest to further explore the direct integration of icebreaking activity through game mechanics. For example, players could be engaged in puzzles that require information exchange for successful completion. Additionally, designers should consider rewarding active icebreaker participation with vanity items that can be used outside of the task, adding value to the icebreaking tasks for players beyond social engagement.

Exemplary game mechanic: A possible implementation in an Online RPG game could be reward mechanisms that are associated with the icebreaking task, e.g., players obtaining collectable tokens through participation. Because RPGs tend to focus on long-term progression, tokens could be collected and then used to purchase vanity items such as costumes or changing spells visual effects. Thereby, the icebreaking task would form part of the game, contribute to progression, and therefore be more likely to be meaningful to players.

\section{$6 \quad$ Limitations and Future Directions}

There are some limitations that need to be considered when interpreting our work. Most importantly, our game RET was designed as a small-scale research tool and therefore only connects three players at a time. Additionally, only a small number of female participants were enrolled in our study, suggesting limited generalizability to women playing games, and raising questions around self-selection. Future work should explore ways of scaling up and connecting larger groups of players to investigate whether and how dynamics change with an increase of player numbers. Likewise, it would be interesting to study the impact of icebreaking tasks in games in an in-the-wild setting to explore how players interact in a non-research context. Moving beyond challenges that the integration of $\mathrm{VC}$ in games poses, exploring opportunities and strengths that are provided by voice communication seems like another valuable avenue: our work suggests that VC does have large potential to bring players together; if designers are looking to build communities, leveraging the effects that $\mathrm{VC}$ has on ties between players could be a valuable design opportunity, for example, by creating games that play with social relationships and directly draw from closeness between players as a game mechanic.

\section{Conclusion}

Voice communication has become an important feature in multiplayer games that is increasingly integrated as a mandatory feature that players are asked to engage with. Our work shows that there are distinct challenges that relate to the revealing nature of $\mathrm{VC}$, suggesting that it may not only contribute to player experience by creating social 
bonds between players, but may also discourage players from further engagement as a result of player discomfort regarding voluntary and accidental sharing of personal information and characteristics (e.g., gender). While our work outlines some opportunities to address player discomfort when first engaging with $\mathrm{VC}$, we need to consider a comprehensive approach to $\mathrm{VC}$ in games that implements mechanisms to reduce discomfort and player harassment. This is particularly important as problems with VC often affect the on-boarding phase of games when players are new to the game, a stage during which player relationships with a game are arguably most fragile and players are most likely to withdraw from a game altogether.

\section{References}

1. Augar, Naomi, Ruth Raitman, and Wanlei Zhou. 2006. Wikis: Collaborative virtual learning environments. In The international handbook of virtual learning environments, pp. 1251-1269.

2. Murray R. Barrick, and Michael K. Mount. 1991. The big five personality dimensions and job performance: a meta_analysis. Personnel psychology 44, no. 1:1-26.

3. Birk, Max, and Regan L. Mandryk. 2013. Control your game-self: effects of controller type on enjoyment, motivation, and personality in game. In Proceedings of the SIGCHI Conference on Human Factors in Computing Systems, pp. 685-694.

4. Blizzard Entertainment. 2016. Video Game. Overwatch.

5. Virginia Braun and Victoria Clarke. 2006. Using thematic analysis in psychology. In Qualitative research in psychology 3, no. 2:77-101.

6. Burgoon, J., Bonito, J., Ramirez, A., Dunbar, N., Kam, K. and Fischer, J. (2002) Testing the interactivity principle: effects of mediation, propinquity, and verbal and nonverbal modalities in interpersonal interaction. Journal of Communication, 52(3) 657-677.

7. Bungie. 2014. Destiny. www.destinythegame.com. last access: 03/04/16.

8. Bohemina Interactive. 2013. DayZ. www.dayzmod.com. last accessed 03/04/16.

9. Marcus Carter, Fraser Allison, John Downs, and Martin Gibbs. 2015. Player Identity Dissonance and Voice Interaction in Games. In Proceedings of the 2015 Annual Symposium on Computer-Human Interaction in Play, 265-269.

10. Marcus Carter, Martin Gibbs, and Greg Wadley. 2013. Death and dying in DayZ. In Proceedings of The 9th Australasian Conference on Interactive Entertainment: Matters of Life and Death, no. 9:22.

11. Depping, Ansgar E., Regan L. Mandryk, Colby Johanson, Jason T. Bowey, and Shelby C. Thomson. 2016. Trust Me: Social Games are Better than Social Icebreakers at Building Trust. In Proceedings of the 2016 Annual Symposium on Computer-Human Interaction in Play.

12. Chlup, Dominique T., and Tracy E. Collins. 2010. Breaking the ice: using ice-breakers and Re-energizers with adult learners. Adult Learning 21.

13. Clear, Tony. 2007. Global virtual teams and 3D collaborative virtual environments. SIENZ'07 - Software Innovation and Engineering New Zealand Workshop 2007, Auckland, New Zealand.

14. Dixon, Julie, Heather Crooks, and Karen Henry. 2006. Breaking the ice: Supporting collaboration and the development of community online. Canadian Journal of Learning and Technology/La revue canadienne de l'apprentissage et de la technologie 32. 
15. Emma F. France, Anne H. Anderson, and Michael Gardner. 2001. The impact of status and audio conferencing technology on business meetings. International Journal of HumanComputer Studies 54, no. 6: 857-876.

16. Frommel, Julian, Katja Rogers, Julia Brich, Daniel Besserer, Leonard Bradatsch, Isabel Ortinau, Ramona Schabenberger, Valentin Riemer, Claudia Schrader, and Michael Weber. 2015. Integrated Questionnaires: maintaining presence in game environments for selfreported data acquisition. In Proceedings of the 2015 Annual Symposium on ComputerHuman Interaction in Play, pp. 359-368.

17. Martin R. Gibbs, Kevin Hew, and Greg Wadley. 2004. Social translucence of the Xbox live voice channel. In Entertainment Computing-ICEC 2004. :377-385.

18. Martin R. Gibbs, Greg Wadley, and Peter Benda. 2004. Proximity-based chat in a first person shooter: using a novel voice communication system for online play. In Proceedings of the 3rd Australasian conference on Interactive entertainment, no. 3:96-102.

19. Samuel D. Gosling, Peter J. Rentfrow, and William B. Swann. 2003. A very brief measure of the Big-Five personality domains. Journal of Research in personality 37, no. 6:504-528.

20. Kevin Hew, Martin R. Gibbs, and Greg Wadley. 2004. Usability and sociability of the Xbox Live voice channel. In Pisan, Y.: Proceedings Australian Workshop on Interactive Entertainment (IE2004), Creative and Cognitive Studios Press, Sydney, :51-58.

21. Jarusriboonchai, Pradthana, Aris Malapaschas, and Thomas Olsson. 2016. Design and Evaluation of a Multi-Player Mobile Game for Icebreaking Activity. In Proceedings of the 2016 CHI Conference on Human Factors in Computing Systems, pp. 4366-4377.

22. Knapp, B., 2002. Ventrilo. Available at www.ventrilo.com

23. Lennart E. Nacke, Chris Bateman, and Regan L. Mandryk. 2011. BrainHex: Preliminary Results from a Neurobiological Gamer Typology Survey. In ICEC, 288-293.

24. Lennart E. Nacke, Chris Bateman, and Regan L. Mandryk. 2014. BrainHex: A neurobiological gamer typology survey. Entertainment computing 5, no. 1:5562.

25. Andreas Löber, Gerhard Schwabe, and Sibylle Grimm. 2007. Audio vs. chat: The effects of group size on media choice. In System Sciences, 2007. HICSS 2007. 40th Annual Hawaii International Conference on, no. 40:41-41.

26. Nasir, Maaz, Kelly Lyons, Rock Leung, and Ali Moradian. 2013. Cooperative games and their effect on group collaboration. In International Conference on Design Science Research in Information Systems, pp. 502-510.

27. Przybylski, Andrew K., C. Scott Rigby, and Richard M. Ryan. 2010. A motivational model of video game engagement. Review of general psychology 14.

28. Rogers, Yvonne, Harry Brignull. 2002. Subtle ice-breaking: encouraging socializing and interaction around a large public display. In Workshop on Public, Community. and Situated Displays.

29. Eva-Lotta, Salinäs. 2002. Collaboration in multi-modal virtual worlds: comparing touch, text, voice and video. In The social life of avatars.

30. Eva-Lotta Sallnäs. 2005. Effects of communication mode on social presence, virtual presence, and performance in collaborative virtual environments. Presence: Teleoperators and Virtual Environments 14, no. 4:434-449.

31. TeamSpeak. 1999. TeamSpeak Communication System. https://www.teamspeak.com/. last accessed 03/04/16.

32. Line E Thomsen, Falko Weigert Petersen, Anders Drachen, and Pejman Mirza-Babaei. 2016. Identifying Onboarding Heuristics for Free-to-Play Mobile Games: A Mixed Methods Approach. In International Conference on Entertainment Computing, pp. 241-246. Springer International Publishing.

33. Tina L. Taylor. 2009. Play between worlds: Exploring online game culture. Mit Press 
34. Tonio Triebel, Benjamin Guthier, Thomas Plotkowiak, and W. Effelberg. 2009. Peer-topeer voice communication for massively multiplayer online games. In Consumer Communications and Networking Conference, 2009. CCNC 2009. 6th IEEE, no. 6:1-5

35. Toups, Zachary O., Andruid Kerne, William A. Hamilton, and Nabeel Shahzad. 2011. Zero-fidelity simulation of fire emergency response: Improving team coordination learning. In Proceedings of the SIGCHI Conference on Human Factors in Computing Systems, pp. 1959-1968.

36. Sherry Turkle. 1995. Life on the screen: identity in the age of the internet. In New York, USA: Simon and Schuster, no. 3:99-100

37. Ubisoft. 2016. The Division. http://tomclancythedivision.ubi.com/game/en-gb/home/. last access 03/04/16.

38. Unity Technologies. 2016. Unity. https://unity3d.com/company.

39. Greg Wadley, Martin R. Gibbs, and Peter Benda. 2005. Towards a framework for designing speech-based player interaction in multiplayer online games. In Proceedings of the second Australasian conference on Interactive entertainment, no. 2:223-226

40. Greg Wadley, Martin Gibbs, and Peter Benda. 2007. Speaking in character: using voiceover-IP to communicate within MMORPGs. In Proceedings of the 4th Australasian conference on Interactive entertainment, no. 4:24

41. Greg Wadley, Martin R. Gibbs, and Nicolas Ducheneaut. 2009. You can be too rich: Mediated communication in a virtual world. In Proceedings of the 21st Annual Conference of the Australian Computer-Human Interaction Special Interest Group: Design: Open 24/7.

42. Greg Wadley, Marcus Carter, and Martin Gibbs. 2015. Voice in virtual worlds: The design, use, and influence of voice chat in online play. Human-Computer Interaction 30.

43. West, Edie. 1999. The big book of icebreakers: quick, fun activities for energizing meetings and workshops. McGraw Hill Professional.

44. Dmitri Williams, Scott Caplan, and Li Xiong. 2007. Can you hear me now? The impact of voice in an online gaming community. In Human communication research 33, no. 4:427449.

45. Dmitri Williams, Nicolas Ducheneaut, Li Xiong, Yuanyuan Zhang, Nick Yee, and Eric Nickell. 2006. From tree house to barracks the social life of guilds in world of warcraft. In Games and culture 1, no. 4:338361.

46. Max L. Wilson, Stuart Reeves, David Coyle, Ed H. Chi. 2014. RepliCHI Workshop II. In Workshop Proceedings of the SIGHCHI Conference on Human Factors in Computing Systems. 